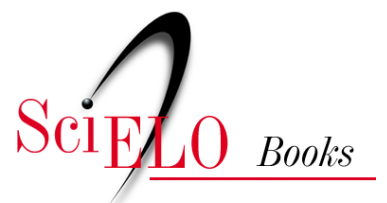

\title{
Capítulo 4 - Controle alternativo de nematoides gastrintestinais de pequenos ruminantes
}

Mário Luan Silva de Medeiros

Larissa Barbosa Nogueira Freitas

Karina Maia Paiva

\section{SciELO Books / SciELO Livros / SciELO Libros}

MEDEIROS, M. L. S., FREITAS, L. B. N., and PAIVA, K. M. Controle alternativo de nematoides gastrintestinais de pequenos ruminantes. In: BEZERRA, A. C. D. S., and SILVA, M. D. C., eds. Fitoterapia e a Ovinocaprinocultura: uma associação promissora [online]. Mossoró: EdUFERSA, 2018, pp. 77-89. ISBN: 978-8557570-91-7. https://doi.org/10.7476/9786587108643.0006.

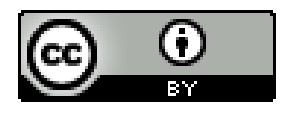

All the contents of this work, except where otherwise noted, is licensed under a Creative Commons Attribution 4.0 International license.

Todo o conteúdo deste trabalho, exceto quando houver ressalva, é publicado sob a licença Creative Commons Atribição 4.0. 


\title{
CONTROLE ALTERNATIVO DE NEMATOIDES GASTRINTESTINAIS DE PEQUENOS RUMINANTES
}

\author{
Mário Luan Silva de Medeiros \\ Larissa Barbosa Nogueira Freitas \\ Karina Maia Paiva
}

\section{INTRODUÇÃO}

A utilização indiscriminada dos anti-helmínticos químicos sintéticos acarretou uma desordem na funcionalidade desses fármacos (em termos de farmacocinética e farmacodinâmica), como também uma nova organização bioquímica e molecular nos nematoides, os quais se tornaram resistentes aos principais grupos químicos de anti-helmínticos existentes (benzimidazóis, imidatiazóis e lactonas macrocíclicas) (MOLENTO, 2004; FORTES; MOLENTO, 2013).

O mecanismo de perda da sensibilidade dos parasitas às classes de químicos sintéticos causa danos irreversíveis nos rebanhos infectados, com consequente aumento de custos no sistema econômico que envolve a ovinocaprinocultura mundial (COLES et al., 2006).

Estratégias alternativas estão sendo desenvolvidas e aplicadas, na perspectiva de promover a prevenção e o tratamento contra nematoides gastrintestinais, buscando principalmente minimizar o uso dos anti-helmínticos sintéticos. Nesse contexto, técnicas adequadas de manejo de animais e de pastagens (HOSTE; TORRES-ACOSTA, 2011), controle biológico dos parasitas (CEZAR et al., 2008), méto- 
dos de desenvolvimento da imunidade animal (RÍOS-DE ÁLVAREZ, 2009), bem como a fitoterapia (HOSTE et al., 2006) são exemplos de estratégias atualmente aplicadas como controle alternativo parasitário. Tais estratégias continuam sendo alvos de pesquisas que visam aperfeiçoa-las para o combate de parasitas nos seus diversos estágios de vida, desde o ovo até o estágio adulto.

\section{ESTRATÉGIAS DE CONTROLE ALTERNATIVO}

\subsection{Manejo de rebanhos e pastagens}

As práticas adotadas de manejo requerem planejamento e visam principalmente à manutenção de um nível seguro de larvas infectantes nas áreas de pastagens, resultando em baixos níveis de helmintoses gastrintestinais em animais de produção, sem causar danos à saúde e à produção desses animais (CEZAR et al., 2008). Algumas técnicas de manejo empregadas no Brasil são: pastejo rotacionado, descontaminação prévia das pastagens e pastejo com alternância de categorias e/ ou espécies de hospedeiros (HOSTE; TORRES-ACOSTA, 2011).

No pastejo rotacionado, a área de pastagem é dividida em piquetes que são utilizados em períodos alternados de pastejo e descanso. Essa técnica tem a vantagem de proporcionar um controle nutricional sobre o pasto e, como consequência, permite a manutenção de um controle parasitário caso o período de pastagem seja inferior ao de desenvolvimento das larvas de helmintos em estágio infectante (L3) (CEZAR et al., 2008). O período de descanso dos piquetes deve durar o suficiente para que as larvas, provenientes das fezes, se tornem inviáveis para causar novas infecções (BARGER, 1999).

Para estabelecer o período de pastejo e descanso, deve-se considerar a influência que as condições climáticas têm sobre o desenvolvimento 
embrionário e larval dos helmintos (CATTO, 1987). Em regiões de clima tropical úmido, a eclosão dos ovos e o desenvolvimento das larvas em L3 ocorrem mais rápido; entretanto, o período de vida da larva infectante é mais curto, pois há um aumento nos seus gastos energéticos, fazendo com que a larva gaste suas reservas mais rapidamente (BARGER, 1999). Assim, em regiões com tais condições climáticas, o tempo de pastejo deve ser curto para evitar a infecção em um mesmo piquete (BARGER, 1999). Em climas temperados, pode demorar cerca de 3 a 9 meses para haver declínio nos níveis de contaminação nos pastos. Isso ocorre porque as fezes infectadas eliminadas no período seco oferecem condições apropriadas para a sobrevivência das larvas infectantes, sendo uma fonte de contaminação na pastagem por até 6 meses, devido à frequência de chuvas (CATTO, 1987).

O processo de descontaminação prévia das pastagens visa a reduzir os riscos de contaminação parasitária, evitando o contato de helmintos gastrintestinais com seus hospedeiros; porém, essa falta ou redução de contato reduz também o desenvolvimento de uma resposta imunológica no animal, especialmente nos animais mais jovens (KUMAR et al., 2012). A descontaminação da pastagem pode ser realizada através de pastejo alternado com diferentes espécies de herbívoros, manutenção do pasto em descanso por um período de tempo que inviabilize os ovos dos helmintos e o desenvolvimento larval, ou ainda adotando-se sistemas de rotação com atividades pecuárias e agrícolas (AMARANTE, 2004).

Os animais encaminhados a essas áreas com pastagens livres de parasitas podem receber pré-tratamento com anti-helmínticos. Essa combinação de estratégias tem como finalidade aumentar ao máximo a produção e reduzir o número de tratamentos com fármacos antihelmínticos (STUEDEMANN et al., 2004). Além disso, é indicado que os animais recém-nascidos sejam transferidos para essas áreas livres de contaminação junto com as fêmeas, logo após o parto, por serem mais susceptíveis a infecções parasitárias (WALLER, 2003). 
No caso do pastejo com alternância de categorias, faixa etária e/ou espécies de hospedeiros, o contato do parasita com o hospedeiro mais susceptível é reduzido (CEZAR et al., 2008). Animais jovens e adultos, quando em um mesmo pasto, reduzem a contaminação do local. Isso ocorre porque os adultos possuem maior imunidade parasitária e, consequentemente, eliminam menor quantidade de ovos nas fezes, reduzindo a concentração de larvas na pastagem (RIBEIRO et al., 2014). Essa alternância da faixa etária mantém a taxa de contaminação do pasto em níveis seguros, porque os animais jovens e adultos competem na ingestão das larvas em estágio L3 no pasto (COLES, 2002).

Na pastagem com diferentes espécies, a redução de infecções é consequência da especificidade parasitária ao hospedeiro. Muitas das espécies de helmintos não parasitam animais de espécies diferentes. Quando as larvas infectantes são ingeridas por um animal que não seja seu hospedeiro preferencial, as chances de infecção são reduzidas (HOSTE; TORRES-ACOSTA, 2011). Quando há infecções parasitárias nessas condições, elas são leves e eliminadas rapidamente pela resposta imunológica do hospedeiro (AMARANTE, 2004).

\subsection{Controle biológico}

Entre as formas de controle alternativo dos nematoides gastrintestinais, destaca-se o uso dos fungos nematófagos e dos besouros coprófagos (CEZAR et al., 2008), organismos conhecidos como antagonistas naturais das infecções quando presentes no ambiente onde se encontram os parasitos infectantes (LARSEN, 2002). Esse controle biológico é uma forma de controle sustentável que, por muitas vezes, reduz a utilização dos produtos químicos nos rebanhos (FONTENOT et al., 2003).

Os principais gêneros de fungos utilizados de modo experimental para o controle de helmintos são: Arthrobotrys, Monacrosporium e 
Duddingtonia, sendo este último o de maior eficácia, quando formulado para essa finalidade, atuando sobre ovos e larvas, principalmente nos primeiros estágios larvais (CEZAR et al., 2008). Assim, há uma redução satisfatória no total de parasitos no ambiente e no sistema gastrintestinal do animal hospedeiro (MOTA et al., 2003).

Apesar dos benefícios e de não haver prejuízo para outros invertebrados continuamente presentes no ambiente, o uso de fungos nematófagos no controle de nematoides gastrintestinais é limitado pela ausência de comercialização significativa dos fungos, pela necessidade do emprego contínuo na dieta dos animais hospedeiros e pela forma de administração em longo prazo (BUSKE et al., 2012; LARSEN, 2002). Portanto, há necessidade de mais estudos e investimentos nesse campo de controle biológico com fungos, com a perspectiva de intensificar a aplicação dessa estratégia de controle.

A utilização de besouros coprófagos para o controle parasitário baseia-se na aplicação desses organismos sobre o material fecal dos animais hospedeiros, onde os besouros podem promover o controle dos nematoides na fase de vida livre e infectante (BERTONE et al., 2005). A espécie Digitonthophagus gazela, por exemplo, tem ação anti-helmíntica cientificamente comprovada; porém a presença de resíduos químicos de anti-helmíntico nas fezes de animais hospedeiros ocasiona efeitos nocivos aos besouros (FLOATE, 2006).

\subsection{Imunidade do hospedeiro}

A presença de provedor imunogênico no organismo dos hospedeiros pode ser essencial e válido para desencadear resposta imunológica contra nematódeos gastrintestinais. Essa característica faz com que os animais respondam positivamente à infecção causada pelos nematoides (MELO; BEVILAQUA, 2005). Tal forma de controle alternativo 
caracteriza-se pelo uso de compostos que promovam ação adjuvante no sistema imune do animal, com o intuito de que a imunidade se torne capacitada a atuar contra os nematoides nocivos ao organismo (RÍOS-DE ÁLVAREZ, 2009). Nesse contexto, estimular o sistema imunológico tornou-se uma alternativa viável no controle parasitário (CEZAR et al., 2008).

Existem algumas estratégias que auxiliam no desenvolvimento imunogênico dos rebanhos. A primeira estratégia caracteriza-se pela seleção genética dos animais já expostos aos nematoides e que desenvolveram no sistema imunológico, a capacidade adaptativa em reconhecê-los e expulsá-los do organismo (VIEIRA, 2005). O método de contagem de ovos por grama de fezes é a principal ferramenta metodológica para verificar essa resposta de resistência nos animais hospedeiros (VANIMISETTI et al., 2004). A ausência de perdas produtivas e de contaminação da pastagem, bem como a diminuição dos custeios são os principais benefícios desse método de controle alternativo (GRAY, 1997). Porém, a estratégia é pouco difundida e há necessidade de mais estudos genéticos que validem a existência da resistência animal aos parasitos (DOMINIK, 2005).

Outra estratégia de controle parasitário baseia-se no uso de determinados compostos na nutrição animal, capazes de desencadear uma melhoria na resposta imune (CEZAR et al., 2008). Uma alimentação constituída de maior carga proteica, por exemplo, pode ser capaz de promover uma diminuição na carga parasitária animal e uma melhor resposta imunológica frente a novas infecções (PHENGVICHITH; LEDIN, 2007). Porém, apesar de comprovado efeito nocivo sobre diversos estágios de vida de parasitos gastrintestinais (RÍOS-DE ÁLVAREZ, 2009), para se fazer uso da suplementação nutricional proteica como estratégia de controle parasitário, ainda são necessárias pesquisas quanto à associação proteína/sensibilidade a nematoides (CEZAR et al., 2008) 


\subsection{Fitoterapia}

A fitoterapia é caracterizada pela utilização de plantas (ou preparações obtidas a partir de plantas) com propriedades medicinais. Baseia-se inicialmente no conhecimento popular para elucidação de espécies vegetais que apresentem potencial para o tratamento de patologias (REZENDE; MONTEIRO-COCCO, 2002). As plantas medicinais apresentam diversas substâncias bioativas responsáveis pelas funções biológicas, sendo utilizadas, por exemplo, como alternativa ao uso de agrotóxicos e anti-helmínticos sintéticos por demonstrarem potencial biológico eficiente e por serem biodegradáveis (CORREA; SALGADO, 2011). Assim, como forma de controle, a fitoterapia é uma alternativa sustentável e eficaz no tratamento de parasitoses e outras doenças animais (LIMA et al., 2012), área de aplicação onde vem ganhando destaque principalmente com o surgimento da resistência aos antiparasitários sintéticos (ALMEIDA et al., 2010).

Pesquisas sobre a atividade biológica de plantas podem ser consideradas promissoras para o desenvolvimento de bioprodutos na forma comercial, pois revelam as potenciais aplicações biotecnológicas de preparações obtidas a partir dessas plantas. Nessa perspectiva, vários autores em todo o mundo têm descrito a atuação de plantas no controle parasitário. No Brasil, as investigações do potencial farmacológico dos fitoterápicos têm revelado espécies biologicamente ativas contra os mais diversos tipos de parasitas, apresentando, por exemplo, ação antiviral (CECÍLIO et al., 2012), antifúngica e leishmanicida (BRAGA et al., 2007), moluscicida e larvicida (LUNA et al., 2005). Embora diversas plantas já tenham sido descritas por suas propriedades medicinais com ação anti-helmíntica, poucas foram validadas cientificamente. No Brasil, em um estudo realizado por KRYCHAK-FURTADO (2006), 106 espécies foram consideradas anti-helmínticas, sendo algumas 
delas utilizadas no tratamento de doenças gastrointestinais causadas por nematoides em pequenos ruminantes.

Em um estudo utilizando teste in vivo em ovinos, tratamentos com Syzygium cumini, Genipa americana e Anacardim humile promoveram significativa atividade ovicida sobre nematoides, comprovada pela redução do número de ovos por grama de fezes (OLIVEIRA, 2013). Em outra pesquisa, extratos aquosos de Mangifera indica apresentaram atividade larvicida sobre Strongyloides stercolaris (EL-SHERBINE, 2014). Foi demonstrado também o efeito anti-helmíntico de extratos aquosos de plantas tropicais, como Gliricidia sepium, Trichanthera gigantean e Leucaena leucocephala, sobre a forma larval (L1) de $H$. contortus (RIOS DE ALVAREZ et al., 2011). No Zimbabwe, pesquisadores revelaram que houve redução significativa no número de ovos em caprinos artificialmente infectados com larvas de H. contortus, quando esses animais foram nutridos com plantas medicinais, como Acacia karoo (KAHIYA et al., 2003).

A validação científica dos fitoterápicos é uma etapa fundamental e necessária para a utilização correta das plantas medicinais e de seus compostos bioativos. Essa validação pode ser realizada pela implementação de testes in vitro com extratos naturais (COSTA et al., 2002), pela caracterização bioquímica dos extratos e pela identificação dos compostos ativos responsáveis por sua ação biológica, objetivando-se aplicar biomoléculas (constituintes desses extratos) como ferramentas biotecnológicas (GARCIA, 1995). 


\section{REFERÊNCIAS}

ALMEIDA, F.A. et al. Multiple resistance to anthelmintics by Haemonchus contortus and Trichostrongylus colubriformis in sheep in Brazil. Parasitology International, v.59, p.622-625, 2010.

AMARANTE, A.F.T. Controle integrado de helmintos de bovinos e ovinos. Revista Brasileira de Parasitologia Veterinária, v.13, supl.1, p.68-71, 2004.

BARGER, I.A. The role of epidemiological knowledge and grazing management for helminth control in small ruminants. International journal for parasitology, v. 29, n. 1, p. 41-47, 1999.

BERTONE, M. et al. Seasonal activity and species composition of dung beetles (Coleoptera: Scarabaeidae and Geotrupidae) inhabiting cattle pastures in North Carolina. Annals of Entomological Society of America, v.98, n.3, p.309-321, 2005.

BRAGA, F.G. et al. Antileishmanial and antifungal activity of plants used in traditional medicine in Brazil. Journal of Ethnopharmacology, v. 111, p. 396-402, 2007.

BUSKE, R. et al. In vitro influence of temperature on the biological control activity of the fungus Duddingtonia flagrans against Haemonchus contortus in sheep. Parasitology Research, February 2013, Volume 112, Issue 2, pp 473-478, 2012.

CATTO, J.B. Longevidade de larvas infectantes de nematódeos gastrointestinais de bovinos no Pantanal Mato-grossense. Pesquisa Agropecuária Brasileira, v. 22, n. 8, p. 847-854, 1987.

CECÍLIO, A.B. et al. Screening of Brazilian medicinal plants for antiviral activity against rotavirus. Journal of Ethnopharmacology, v. 141, p. 975-981, 2012.

CEZAR, A.S.; CATTO, J.B.; BIANCHIN I. Controle alternativo de nematódeos gastrintestinais dos ruminantes: atualidade e perspectivas. Ciência Rural, v. 38, n. 7, 2008. 
COLES, G.C. Cattle nematodes resistant to anthelmintic: why so few cases? Veterinary Research, v.33, p.481-489, 2002.

COLES, G.C. et al. The detection of anthelmintic resistance in nematodes of veterinary importance. Veterinary Parasitology, v.136, p.167-185, 2006.

CORREAA, J.C.R.; SALGADO, H.R.N. Atividade inseticida das plantas e aplicações: revisão. Revista Brasileira de Plantas Medicinais, v.13, p.500 -506, 2011.

COSTA. C.T.C. et al. Efeito ovicida de extratos de sementes de Mangifera indica L. sobre Haemonchus contortus. Revista Brasileira de Parasitologia Veterinária. v. 11,p. 57-60, 2002.

DOMINIK, S. Quantitative trait loci for internal nematode resistance in sheep: a review. Genetics Selection Evolution, v.37, supl.1, p. S83-S96, 2005.

EL-SHERBINI, G.T. Anthelmintic activity of unripe Mangifera indica L. (Mango) against Strongyloides stercolaris. International Journal of Medicinal Plant and Alternative Medicine. v. 1, n.4, p. 73-79, 2014.

FLOATE, K.D. Endectocide use in cattle and fecal residues: environmental effects in Canada. Canadian Journal of Veterinary Research, v.70, p.1-10, 2006.

FONTENOT, M.E. et al. Efficiency of feeding Duddingtonia flagrans chlamydospores to grazing ewes on reducing availability of parasitic nematode larvae on pasture. Veterinary Parasitology, v.118, p.203213, 2003.

FORTES, F.S.; MOLENTO, M.B. Anthelmintic resistance in gastrointestinal nematodes of small ruminants: advances and limitations for diagnosis. Pesquisa Veterinária Brasileira, v.33, n.12, p.1391-1402, 2013.

GARCIA, E.S. Biodiversidade, Biotecnologia e Saúde. Caderneta de Saúde Pública, v.11, n.3, p.491-494, 1995. 
GRAY, G. D. The use of genetically resistant sheep to control nematode parasitism. Veterinary Parasitology, v.72, p.345- 366, 1997.

HOSTE, H. et al. The effects of tannin-rich plants on parasitic nematodes in ruminants. TRENDS in Parasitology, v.22, n.6, p.253261, 2006.

HOSTE, H.; TORRES-ACOSTA, J.F.J. Non chemical control of helminthes in ruminants: adapting solutions for changing worms in a changing world. Veterinary parasitology, v. 180, n. 1, p. 144-154, 2011.

KAHIYA, C.; MUKARATIRWA, S.; THAMSBORG, S.M. Effects of Acacia nilotica and Acacia karoo diets on Haemonchus contortus infection in goats. Veterinary Parasitology, v.115, p.265-74, 2003.

KRYCHAK-FURTADO, S. Alternativas fitoterápicas para o controle da verminose ovina no estado do Paraná: testes in vitro e in vivo. 2006. 147p.

KUMAR, N. et al. Internal parasite management in grazing livestock. Journal of Parasitic Diseases, v. 37, n. 2, p. 151-157, 2012.

LARSEN, M. Biological control in a global perspective - a review with emphasis on Duddingtonia flagrans. In: Biological control of nematode parasites of small ruminants in Asia. Final proceeding. FAO, Animal Production and Health Division, Rome, Italy, 104p. (FAO Animal Production and Health Paper), 2002.

LIMA, R.P. et al. Emprego de plantas medicinais em animais de companhia e de produção da zona rural do município de Juru-PB. Revista de Biologia e Farmácia, v.08, p.85-92, 2012.

LUNA, J.S. et al. A study of the larvicidal and molluscicidal activities of some medicinal plants from northeast Brazil. Journal of Ethnopharmacology, v. 97, p. 199-206, 2005.

MELO, A.C.F.L.; BEVILAQUA, C.M.L. Genetic approach of anthelmintic resistance in Haemonchus contortus. Revista Portuguesa de Medicina Veterinária, v.100, p. 141-146, 2005. 
MOLENTO, M. B. Resistência de helmintos em ovinos e caprinos. Revista Brasileira de Parasitologia Veterinária, v.13, supl.1, p.8287, 2004.

MOTA, M.A.; CAMPOS, A.K.; ARAUJO, J.V. Controle biológico de helmintos parasitos de animais: estágio atual e perspectivas futuras. Pesquisa Veterinária Brasileira, v.23, n.3, p.93-100, 2003.

OLIVEIRA, L.D.R. Plantas medicinais como alternativa para o controle de Haemonchus contortus em ovinos: testes in vitro e in vivo. 74f. Dissertação (Mestrado em Ciências Animais), Faculdade de Agronomia e Medicina Veterinária da Universidade de Brasília, 2013.

PHENGVICHITH, V.; LEDIN, I. Effect of a diet high in energy and protein on growth, carcase characteristics and parasite resistance in goats. Tropical Animal Health and Production, n.39, p.59-70, 2007.

REZENDE, H. A.; MONTEIRO-COCCO, M. I. The Phytoterapy Utilization in the Rural Population Routine. Revista Escola Enfermagem., v. 3, n. 36, p.282-288, 2002.

RIBEIRO, C.M. et al. Susceptibilidade à infecção por helmintos gastrintestinais em bovinos leiteiros da mesorregião do sudoeste paranaense, Brasil. Veterinária e Zootecnia, v. 21, n. 1, p. 154-159, 2014.

RÍOS-DE ÁLVAREZ, L. Mechanisms of action of plant secondary metabolites and their effect on the imune response of parasitised sheep. Thesis (Doctor of Philosophy), University of Edinburgh, $\mathrm{f}$. 189. 2009.

STUEDEMANN, J.A. et al. Bermudagrass management in the Southern Piedmont USA. V: Gastrointestinal parasite control in cattle. Veterinary Parasitology, v.126, p.375-385, 2004.

VANIMISETTI, H.B. et al. Inheritance of fecal egg count and packed cell volume and their relationship with production traits in sheep infected with Haemonchus contortus. Journal of Animal Science, v.82, p.1602-1611, 2004. 
VIEIRA, L. S. Endoparasitoses gastrintestinais em caprinos e ovinos. Sobral : Embrapa Caprinos, 32 p., 2005 (Série Documentos / Embrapa Caprinos, ISSN 1676-7659; 58).

WALLER, P.J. Global perspectives on nematode parasite control in ruminant livestock: the need to adopt alternatives to chemotherapy, with emphasis on biological control. Animal Health Research Reviews, v. 4, n. 01, p. 35-44, 2003. 